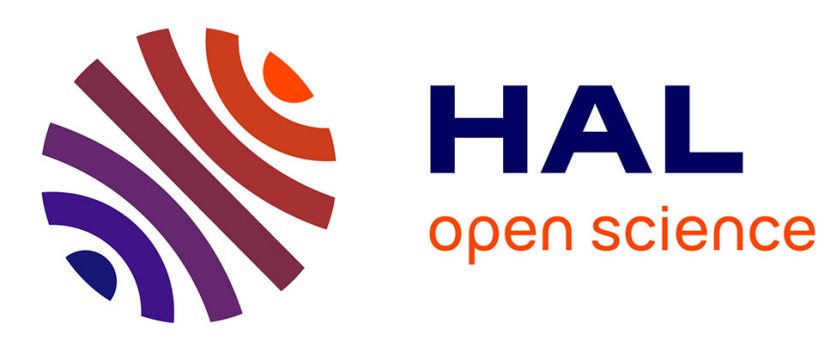

\title{
Asymptotic modeling of quasi-brittle interfaces
}

Céline Pelissou, Frédéric Lebon

\section{To cite this version:}

Céline Pelissou, Frédéric Lebon. Asymptotic modeling of quasi-brittle interfaces. Computers \& Structures, 2009, 87 (19-20), pp.1216-1223. 10.1016/j.compstruc.2008.12.002 . hal-00459498

\section{HAL Id: hal-00459498 https://hal.science/hal-00459498}

Submitted on 1 Jun 2018

HAL is a multi-disciplinary open access archive for the deposit and dissemination of scientific research documents, whether they are published or not. The documents may come from teaching and research institutions in France or abroad, or from public or private research centers.
L'archive ouverte pluridisciplinaire HAL, est destinée au dépôt et à la diffusion de documents scientifiques de niveau recherche, publiés ou non, émanant des établissements d'enseignement et de recherche français ou étrangers, des laboratoires publics ou privés. 


\title{
Asymptotic modeling of quasi-brittle interfaces
}

\author{
Céline Pelissou ${ }^{\mathrm{a}, \mathrm{b}}$, Frédéric Lebon ${ }^{\mathrm{a}, *}$ \\ ${ }^{a}$ LMA UPR-CNRS 7051, Aix-Marseille University, 13402 Marseille Cedex 20, France \\ b IRSN, CE Cadarache, BP3, 13115 St. Paul-lez-Durance Cedex, France
}

\begin{abstract}
This study deals with the damage modeling of quasi-brittle interfaces such as the mortar/brick interfaces present in masonry walls. For this purpose, a model is developed based on a bulk model presented by Gambarotta and Lagomarsino, which takes the damage to the mortar joint into account. A quasi-fragile damage interface model is introduced using an asymptotic technique. This model memorizes some of the geometrical and mechanical characteristics of the interface, such as the thickness, elastic coefficients, normal and tangential stress, and the damage variable. Numerical simulations are performed using the Gyptis finite element software: academic cases involving traction and shear loads are presented.
\end{abstract}

\section{Introduction}

The modeling of interfaces between solids in structure assemblies is of great importance in mechanical technology. These interfaces contribute crucially to the strength of many structures. It is therefore necessary to develop rather fine models. One of the main problems arising here is that of developing a unified theory to model interfaces, since the contact is often unique from a tribological point of view, i.e. the model depends on the materials in contact. Another problem is due to the smallness of the interface as well as its low rigidity. A large class of models has been developed in previous studies to describe the behavior of interfaces (see [20] and the references therein). Many studies based on the use of asymptotic techniques have focused on the behavior of interfaces (see for example $[8,16,10,13,14,12]$ and the references therein). The aim of these techniques is to take microscopic details into account in the interface model. In the present study, we are especially interested in defining the behavior of quasi-brittle interfaces. A new model is presented for simulating the damage occurring in interfaces of this kind, using an asymptotic technique. This modeling procedure takes into account mechanical processes responsible for rupture, such as the unilateral contact and friction on the microscopic scale and the normal and tangential damage. The model was implemented in a finite element software and tested successfully on some academic examples.

\footnotetext{
* Corresponding author.

E-mail addresses: celine.pelissou@irsn.fr (C. Pelissou), lebon@lma.cnrs-mrs.fr (F. Lebon).
}

This paper consists of four parts. In the first part, previous experimental results are recalled. The second part is devoted to the modeling of quasi-brittle interfaces; the modeling procedure is decomposed into three steps. In the third part, the numerical implementation in a finite element software is presented; two algorithms are described in detail. The fourth part gives the numerical results obtained in the case of two examples.

\section{Characterization of interfaces: comments on experimental results}

In a former study, we attempted to characterize the local behavior of the interface between full and hollow bricks and mortar joints, which constitutes a typical quasi-brittle interface (see $[4,5])$. The results obtained are summarized below.

The experimental device used $[2,3]$ was designed for studying on the local scale the shearing behavior of a simple assembly of two (Fig. 1) and three (Fig. 2) full or hollow bricks $(210 \times 50 \mathrm{~mm})$ which are connected by a mortar joint $10 \mathrm{~mm}$ thick. The samples are subjected to a monotonously increasing load up to rupture.

We observed the occurrence of the following behavior (see also Fig. 3):

- rigid elastic behavior up to the rupture, followed by friction sliding;

- the behavior of full bricks is fragile after the limit strength has been reached;

- the behavior of hollow bricks is quasi-fragile after the limit strength has been reached; 


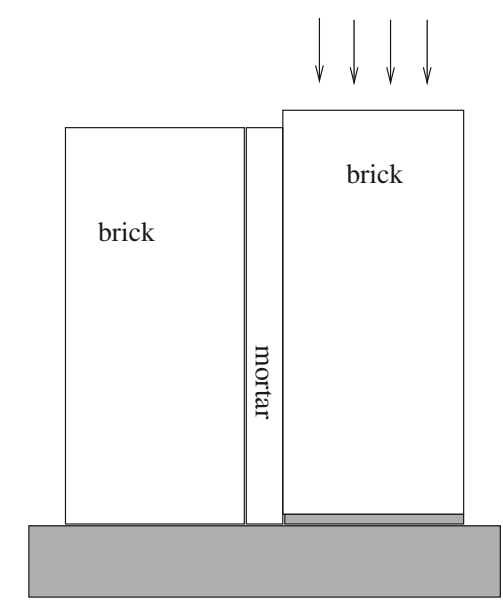

Fig. 1. Experimental device with two bricks.

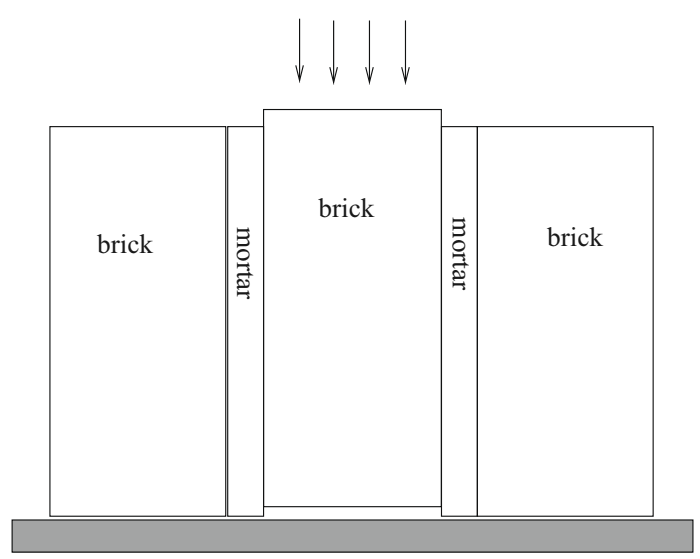

Fig. 2. Experimental device with three bricks.

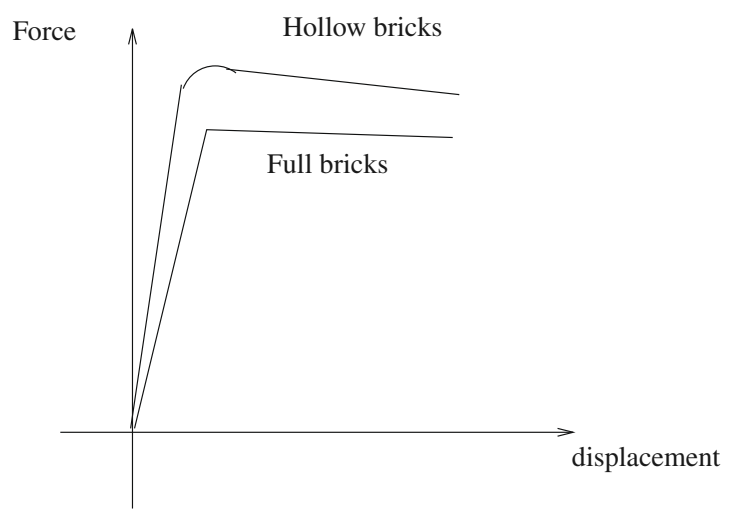

Fig. 3. (Dimensionless) Typical behavior of the mortar/brick interface (full and hollow bricks).

- wide dispersion of the data in the case of hollow brick samples, due mainly to the non-uniform distribution of the mortar spikes and local defects in the components of the bricks;

- two and three bricks samples show similar behavior. The choice of the basic cell therefore has no effect on the local scale.

\section{Model description}

\subsection{General considerations and notations}

The model described in this section is an extension of the bulk model introduced by Gambarotta and Lagomarsino [6], which takes the damage of the mortar joint into account. The interface modeling procedure (Fig. 4) used consists of three steps:

- We consider a macroscopic bulk model for quasi-fragile materials.

- We assume the structure to consist of three phases: material 1 (brick for example), material 2 (mortar for example) and a thin interphase between the two materials, formed by the material described in the first step.

- Since the interphase is thin, we obtain an interface model by performing an asymptotic analysis [7,15], where the thickness of the interface tends to zero.

The model obtained memorizes some of the geometrical and mechanical characteristics of the interphase such as its thickness, elastic coefficients, normal and tangential stress, and the damage variable.

For the sake of simplification, we consider a structure occupying an open bounded set $\Omega$ of $\mathbb{R}^{2}$, with a smooth boundary $\partial \Omega$. The twodimensional space is referred to the orthonormal frame $\left(0, x_{1}, x_{2}\right)$.

\subsection{First step: bulk model}

In this section, the bulk model introduced by Gambarotta and Lagomarsino [6] is briefly described. This model is dedicated to masonry structures: the masonry is taken to be a "material" showing non-linear damage behavior. The macroscopic behavior is determined by applying an averaging process based on microscopic considerations. As classically done, the strain tensor is decomposed into linear and non-linear (anelastic) parts:

$\varepsilon=\varepsilon^{e}+\varepsilon^{a n}$

with

$\sigma=K \varepsilon^{e} \quad$ and $\quad \varepsilon^{a n}=S_{\sigma} \sigma$

where $K$ denotes the elastic rigidity tensors. At a local level (on a Representative Elementary Volume, Fig. 5), the stress vector is decomposed into normal $\sigma_{n}$ and tangential $\tau$ parts and the normal and tangential components of $\boldsymbol{\varepsilon}^{a n}$ are expressed by (3):

$\left\{\begin{array}{l}\varepsilon_{n}^{a n}=h \alpha H\left(\sigma_{n}\right) \sigma_{n} \\ \varepsilon_{t}^{a n}=k \alpha(\tau-f)\end{array}\right.$

where $f=\tau$ if $\tau \in I=]-\mu \sigma_{n},+\mu \sigma_{n}$ [ and $f= \pm \mu \sigma_{n}$ if $\tau \notin I$ (Fig. 6). $H$ is the Heaviside function of the unilateral response of the joint and $\alpha$ is the damage variable. $h$ and $k$ are positive coefficients denoting the opening and the sliding compliances of the mortar joint, and $\mu$ is the internal friction coefficient.

We can write

$\varepsilon_{t}^{a n}=k \alpha \chi_{I}(\tau)\left(\tau \pm \mu \sigma_{n}\right)$

where $\chi_{I}$ is the characteristic function of the set $\bar{I}$, complementary set of $I$, such that:

- If $\tau \notin I, \chi_{I}(\tau)=1$, there is tangential damage.

- If $\tau \in I, \chi_{I}(\tau)=0$, there is no tangential damage.

$S_{\sigma}$ is therefore given locally in matrix form by:

$S_{\sigma}=\left(\begin{array}{cc}h \alpha H\left(\sigma_{n}\right) & 0 \\ \pm k \alpha \chi_{I}(\tau) \mu & k \alpha \chi_{I}(\tau)\end{array}\right)$ 


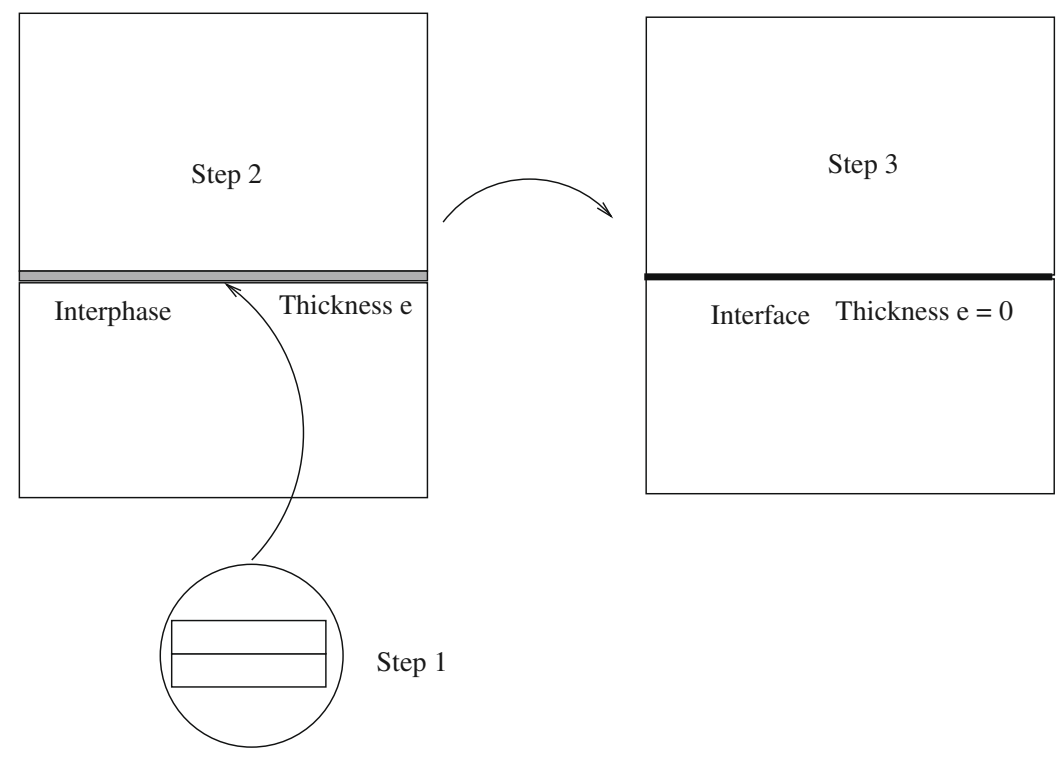

Fig. 4. Three steps interface modeling procedure.

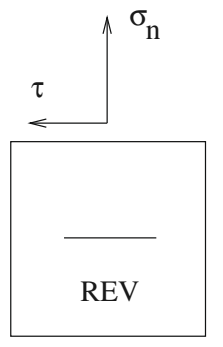

Fig. 5. Normal and tangential stress vector components.

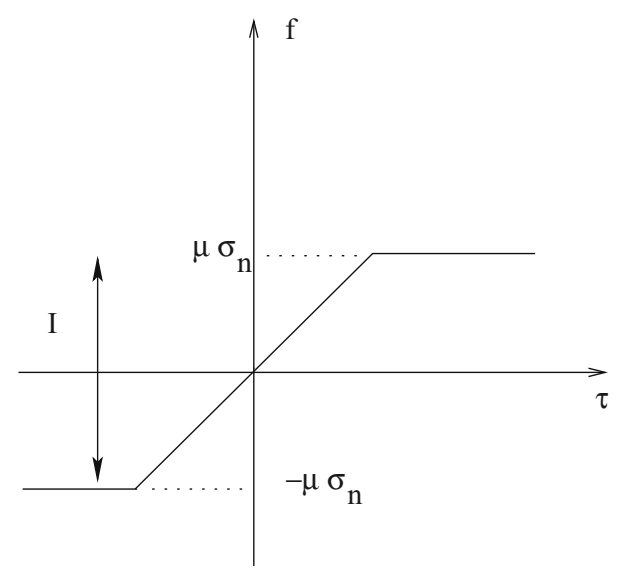

Fig. 6. Relation between $f$ and $\tau$ (friction threshold).

The damage variable $\alpha$ is governed by a yield condition:

$\left\{\begin{array}{l}\Phi(\alpha)<0 \text { no evolution of the damage } \\ \Phi(\alpha)=0 \text { evolution of the damage }\end{array}\right.$

where

$\Phi(\alpha)=Y-R(\alpha)$

$Y=\frac{1}{2} h H\left(\sigma_{n}\right) \sigma_{n}^{2}+\frac{1}{2} k \chi_{I}(\tau)\left(\tau \pm \mu \sigma_{n}\right)^{2} \quad$ (energy release rate)

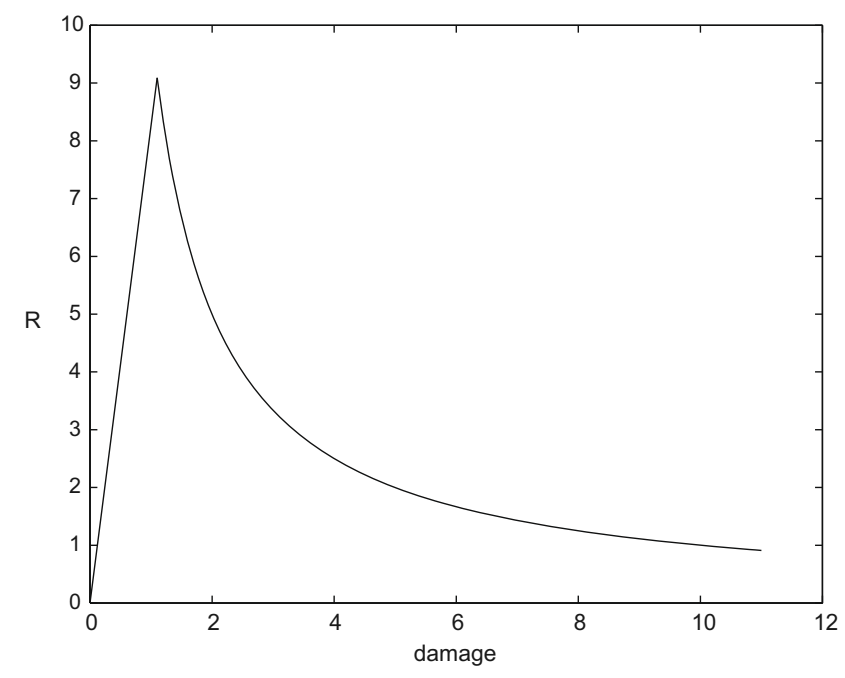

Fig. 7. Evolution of $R(\alpha)$.

$R$, the material toughness, is defined by (see Fig. 7):

$$
R(\alpha)= \begin{cases}R_{0} \alpha & \text { if } 0 \leqslant \alpha \leqslant 1 \\ \frac{R_{0}}{\alpha} & \text { if } \alpha \geqslant 1\end{cases}
$$

The parameter $R_{0}$ is a material coefficient of toughness.

\subsection{Second step: the interphase}

In this section, the structure is taken to consist of two materials separated by a thin interphase which is a "mixture" of the other two materials. The interphase is assumed to be parallel to the $x_{1}$ axis and the thickness, which is assumed to be constant, is denoted by $e$. In what follows, the constitutive equations are those given in the previous section, i.e. the interface consists of a quasi-brittle material. We assume the elastic part to be isotropic and taking the external normal vector $x_{2}$, the constitutive equation is $\sigma=\left(I_{d}+K S_{\sigma}\right)^{-1} K \varepsilon=K_{\sigma} \varepsilon$

$I_{d}$ is the identity tensor of order two. 
Introducing the Lame's coefficients $\lambda$ and $G$, we can write:

$$
K_{\sigma}=\left(\begin{array}{ccc}
\lambda+2 G-\frac{\lambda^{2} h \alpha H}{1+(\lambda+2 G) h \alpha H} & \lambda-\frac{\lambda(\lambda+2 G) h \alpha H}{1+(\lambda+2 G) h \alpha H} & 0 \\
\frac{\lambda}{1+(\lambda+2 G) h \alpha H} & \frac{\lambda+2 G}{1+(\lambda+2 G) h \alpha H} & 0 \\
-\frac{ \pm \mu \lambda G k \alpha \chi_{I}}{(1+(\lambda+2 G) h \alpha H)\left(1+G k \alpha \chi_{I}\right)} & -\frac{ \pm \mu(\lambda+2 G) G k \alpha \chi_{I}}{(1+(\lambda+2 G) h \alpha H)\left(1+G k \alpha \chi_{I}\right)} & \frac{G}{1+G k \alpha \chi_{I}}
\end{array}\right)
$$

\subsection{Third step: asymptotic expansions}

\subsubsection{General considerations}

The idea of using matched asymptotic expansions $[1,19]$ is to find two expansions of the displacement $u^{e}$ and of the stress $\sigma^{e}$ in powers of $e$, that is, an external one in the bodies and an internal in the joint, and to connect these two expansions in order to obtain the same limit. In what follows, we have addressed a two-dimensional problem in order to simplify the computations. The relations obtained in the internal expansions will be expressed using values occurring in the external expansions.

\subsubsection{External expansions}

The external expansion is a classical expansion in powers of $e$ :

$u^{e}\left(x_{1}, x_{2}\right)=u^{0}+e u^{1}+\cdots+e^{n} u^{n}+\cdots$

$\varepsilon_{i j}\left(u^{e}\right)\left(x_{1}, x_{2}\right)=\varepsilon_{i j}^{0}+e \varepsilon_{i j}^{1}+\cdots+e^{n} \varepsilon_{i j}^{n}+\cdots$

$\left.\varepsilon_{i j}^{l}=\frac{1}{2} \frac{\partial u_{i}^{l}}{\partial x_{j}}+\frac{\partial u_{j}^{l}}{\partial x_{i}}\right)$

$\sigma_{i j}^{e}\left(x_{1}, x_{2}\right)=\sigma_{i j}^{0}+e \sigma_{i j}^{1}+\cdots+e^{n} \sigma_{i j}^{n}+\cdots$

$u^{n}=u^{n}\left(x_{1}, x_{2}\right), \varepsilon_{i j}^{n}=\varepsilon_{i j}^{n}\left(x_{1}, x_{2}\right)$ and $\sigma_{i j}^{n}=\sigma_{i j}^{n}\left(x_{1}, x_{2}\right)$ are the coefficients of the expansions.

\subsubsection{Internal expansions}

In the internal expansion, we perform a blow-up of the second variable. Let us denote $y_{2}=\frac{x_{2}}{e}$. The internal expansion gives:

$$
\begin{aligned}
& u^{e}\left(x_{1}, x_{2}\right)=v^{0}+e v^{1}+\cdots+e^{n} v^{n}+\cdots \\
& \varepsilon_{i j}\left(u^{e}\right)\left(x_{1}, y_{2}\right)=e^{-1} \varepsilon_{i j}^{-1}+\varepsilon_{i j}^{0}+e \varepsilon_{i j}^{1}+\cdots+e^{n} \varepsilon_{i j}^{n}+\cdots \\
& \varepsilon_{11}^{l}=\frac{\partial v_{1}^{l}}{\partial x_{1}} \\
& \varepsilon_{22}^{l}=\frac{\partial v_{2}^{l+1}}{\partial y_{2}} \\
& \varepsilon_{12}^{l}=\frac{1}{2}\left(\frac{\partial v_{2}^{l}}{\partial x_{1}}+\frac{\partial v_{1}^{l+1}}{\partial y_{2}}\right) \\
& \sigma_{i j}^{e}\left(x_{1}, x_{2}\right)=e^{-1} \tau_{i j}^{-1}+\tau_{i j}^{0}+e \tau_{i j}^{1}+\cdots+e^{n} \tau_{i j}^{n}+\cdots \\
& \left.\sigma_{i j, j}^{e}=\sum_{l=-2}^{\infty} e^{l} \frac{\partial \tau_{i 1}^{l}}{\partial x_{1}}+\frac{\partial \tau_{i 2}^{l+1}}{\partial y_{2}}\right) .
\end{aligned}
$$

We use the convention $\quad v^{l}=0$ if $l<0, \quad \tau^{l}=0$ if $l<-1$. $v^{n}=v^{n}\left(x_{1}, y_{2}\right)$ and $\tau_{i j}^{n}=\tau_{i j}^{n}\left(x_{1}, y_{2}\right)$ are the coefficients of the expansions.

\subsubsection{Continuity conditions}

The third step in the method consists in connecting of the two expansions. We choose some intermediate lines defined by $\left.x_{2}= \pm \theta \varepsilon^{t}, 0<t<1, \theta \in\right] 0,+\infty\left[\right.$. When $e$ tends to zero, $x_{2}$ tends to $0^{ \pm}$and $y_{2}=x_{2} / e$ tends to $\pm \infty$. The principle of the method [1,19] consists in assuming that the two expansions give the same asymptotic limits, that is (i) $v^{0}\left(x_{1}, \pm \infty\right)=u^{0}\left(x_{1}, 0^{ \pm}\right)$

(ii) $\tau^{-1}\left(x_{1}, \pm \infty\right)=0$

(iii) $\tau^{0}\left(x_{1}, \pm \infty\right)=\sigma^{0}\left(x_{1}, 0^{ \pm}\right)$

\subsubsection{Interface behavior}

Using this asymptotic approach (which is similar to that used in [15] in a different context), by injecting the asymptotic expansions into the constitutive equations and in the equilibrium equations, we deduce expressions linking the stress vector to the jump in the displacement denoted by $[u]$ :

$\tau_{i j}^{-1}=0$

$v_{j}^{0}\left(x_{1}, \pm\left|y_{2}\right|\right)=u_{j}^{0}\left(x_{1}, 0^{ \pm}\right)$

$\tau_{i 2}^{0}\left(x_{1}, \pm\left|y_{2}\right|\right)=\sigma_{i 2}^{0}\left(x_{1}, 0\right)$

We obtain

$$
\begin{aligned}
\tau_{22}^{0}= & \lim _{e \rightarrow 0} \frac{\lambda+2 G}{1+(\lambda+2 G) h \alpha H} \frac{\partial v_{2}^{0}}{\partial y_{2}} \\
\tau_{12}^{0}= & \lim _{e \rightarrow 0} \frac{ \pm \mu(\lambda+2 G) G k \alpha \chi_{I}}{(1+(\lambda+2 G) h \alpha H)\left(1+G k \alpha \chi_{I}\right)} \frac{\partial v_{2}^{0}}{\partial y_{2}} \\
& +\lim _{e \rightarrow 0} \frac{G}{1+G k \alpha \chi_{I}} \frac{\partial v_{1}^{0}}{\partial y_{2}}
\end{aligned}
$$

Thus, by integration, the Asymptotic Gambarotta-Lagomarsino (A$\mathrm{GL}$ ) model is written in terms of the following system of normal and tangential components:

$$
\left\{\begin{array}{l}
\sigma_{n}=\frac{C_{N}}{1+C_{N} h \alpha H\left(\sigma_{n}\right)}\left[u_{N}\right] \\
\tau=\frac{ \pm C_{N} C_{T} \mu \bar{k} \alpha \chi_{I}}{\left(1+C_{N} h \alpha H\left(\sigma_{n}\right)\right)\left(1+C_{T} k \alpha \chi_{I}\right)}\left[u_{N}\right]+\frac{C_{T}}{1+C_{T} k \alpha \chi_{I}}\left[u_{T}\right]
\end{array}\right.
$$

where

$$
\begin{aligned}
& C_{N}=\lim _{e \rightarrow 0}(\lambda+2 G) / e \\
& C_{T}=\lim _{e \rightarrow 0} G / e \\
& \bar{h}=\lim _{e \rightarrow 0} h e \\
& \bar{k}=\lim _{e \rightarrow 0} k e
\end{aligned}
$$

The resulting matrix is not diagonal: a non-symmetric coupling term appears between the normal compliance term $C_{N}$ and the tangential compliance term $C_{T}$. It is worth noting that in this general expression, expressions obtained under traction conditions when $f=0$ are combined with those obtained under compression when $f=\tau$ and when the friction threshold is reached with $f= \pm \mu \sigma_{n}$.

The damage is governed by the following yield condition:

$\bar{\Phi}(\alpha)=\bar{Y}-\bar{R}(\alpha) \leqslant 0$

with

$$
\left\{\begin{array}{l}
\bar{Y}=\frac{1}{2} \bar{h} H\left(\sigma_{n}\right) \sigma_{n}^{2}+\frac{1}{2} \bar{k} \chi_{I}(\tau)\left(\tau- \pm \mu \sigma_{n}\right)^{2} \\
\bar{R}(\alpha)=\lim _{e \rightarrow 0} R e= \begin{cases}\bar{R}_{0} \alpha & \text { if } 0<\alpha<1 \\
\frac{\bar{R}_{0}}{\alpha} & \text { if } \alpha>1\end{cases}
\end{array}\right.
$$

Comment: The asymptotic expansion technique is formal from a mathematical point of view. However, in former works, it is shown rigorously (by variational methods) [12] or numerically [14], that the results obtained using this technique, are relevant.

\section{Numerical modeling}

In this part, we describe the numerical modeling procedure used for our asymptotic scheme. The finite element method is used to perform the spatial discretization. Here we present the condensed 
spatial discretization system obtained, the damage condition introduced into the model, and two algorithms for solving it.

\subsection{Spatial discretization}

Let us briefly recall the weak formulation of a standard elastic problem, having the following form:

$\int_{\Omega} A \varepsilon(u) \cdot \varepsilon(v) d \Omega-\int_{\Gamma_{s}} C[u] \cdot[v] d \Gamma=\int_{\Omega} f v d \Omega+\int_{\Omega} s \cdot v d \Gamma$

where $f$ and $s$ denote the volume and the surface forces, respectively, $\Gamma_{S}$ is part of the boundary $\partial \Omega$. $A$ is the fourth order elasticity tensor, and $C$ is the non-symmetric stiffness matrix and depends on the damage with:

$C=\left(\begin{array}{cc}C_{x x}(\alpha) & 0 \\ C_{y x}(\alpha) & C_{y y}(\alpha)\end{array}\right)$

Writing the displacement jump $[u]=N \delta[u]$, the discretization of the surface term is obtained as follows:

$$
\begin{aligned}
\int_{\Gamma_{s}} C[u] \cdot[v] d \Gamma & =\sum_{\text {seg }} \int_{\text {seg }} \delta[v] N^{t} C N \delta[u] d x \\
& =\sum_{\text {seg }} \int_{\text {seg }} \delta[v] \widetilde{V} \delta[u] d x
\end{aligned}
$$

A linear interpolation is performed (cf. Fig. 8a), taking $x$ to denote the abscissa associated with the segment of length $h . V^{e l}$ is the order 4 elementary matrix associated with the length of the segment $h$,

$V^{e l}=\int_{0}^{h} \widetilde{V} d x=\int_{0}^{h} N^{t} C N d x$

After proceeding assembling the matrix, we then obtain the linear system where $K$ and $V$ are the rigidity matrix and the matrix associated with the damage interface, respectively.

$K \delta u-V \delta[u]=F \quad$ for a given $\alpha$

\subsection{The condensed damage problem}

The first step in the process consists in condensing the mechanical problem (25) on the contact surfaces (between the two solids) as follows:

$$
\left(\begin{array}{ll}
K_{l l} & K_{l c} \\
K_{c l} & K_{c c}
\end{array}\right)\left(\begin{array}{l}
u_{l} \\
u_{c}
\end{array}\right)+\left(\begin{array}{cc}
0 & 0 \\
0 & V_{c c}
\end{array}\right)\left(\begin{array}{l}
{\left[u_{l}\right]} \\
{\left[u_{c}\right]}
\end{array}\right)=\left(\begin{array}{l}
F_{l} \\
F_{c}
\end{array}\right)
$$

Hence

$$
\begin{aligned}
& {\left[K_{c c}-K_{c l} K_{l l}^{-1} K_{l c}\right] u_{c}+V_{c c}\left[u_{c}\right]=F_{c}-K_{c l} K_{l l}^{-1} F_{l}} \\
& A_{c c} u_{c}+\widetilde{A}_{c c}\left[u_{c}\right]=\tilde{F}_{c} \quad \text { with } u_{i}=\left\{u_{N i}, u_{T i}\right\}^{t}
\end{aligned}
$$

The indexes $c$ and $l$ correspond here to the contact nodes and the free nodes (see Fig. 9). The rigidity matrix is then decomposed into two terms:

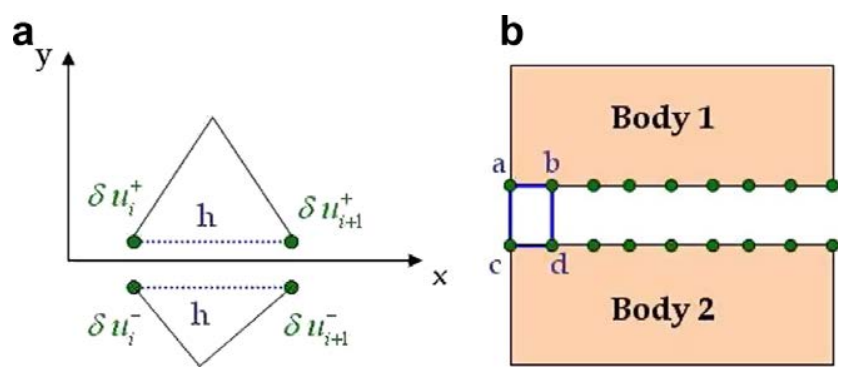

Fig. 8. (a) Displacement jump; (b) quadrangular element at the interface.

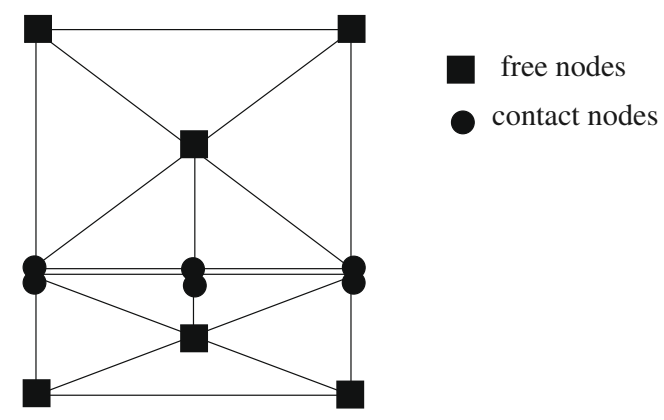

Fig. 9. Example of free and contact nodes.

- The first one is constant: it is the bulk matrix $A_{c c}$.

- The second one depends on the damage interface: it is the non-symmetric matrix $\widetilde{A}_{c c}$, with the same order as $A_{c c}$.

\subsubsection{Choice of quadrangular elements}

Here it is proposed to deal with the damage phenomena occurring at the interface between two solids. Taking $a$ to denote the contact node of body 1 , we find its associated quadrangle (cf. Fig. $8 \mathrm{~b}$ ). This element is formed by the neighboring node $b$ and the two opposite nodes on the body 2, $c$ and $d$. For the spatial discretization, we therefore introduced a virtual connectivity via these quadrangles, in order to define the damage occurring at the interface, which is given by the surface term (23).

\subsubsection{Two types of contact}

Two types of contact are studied: a "deformable" contact between two deformable bodies (Fig. 10a) and a "rigid" contact between a deformable body and a rigid body (Fig. 10b).

The condensed matrix $A_{c c}$ therefore has a diagonal matrix form consisting of blocks:

$A_{c c}=\left(\begin{array}{cc}A_{c c}^{1} & 0 \\ 0 & A_{c c}^{2}\end{array}\right) A_{c c}^{1}$ and $A_{c c}^{2}$ in relation to body 1 and body 2,

respectively. The condensed damage matrix $\widetilde{A}_{c c}$ is deduced by globally assembling the $(8 \times 8)$ matrix $J$ :

$J=\left(\begin{array}{cc}V^{e l} & -V^{e l} \\ -V^{e l} & V^{e l}\end{array}\right)$ is obtained on each quadrangle from the $(4 \times 4)$ elementary matrix $V^{e l}$ associated with the same quadrangle.

\subsection{The damage condition}

In this section, we explain how the damage condition (19) is handled in keeping with the previous damage value (making the distinction between $\alpha<1$ and $\alpha>1$ ), under pure traction or pure sliding or a combination of traction/sliding conditions. We introduce two new notations:

$h_{N}=C_{N} \bar{h} \quad$ and $\quad h_{T}=C_{T} \bar{k}$
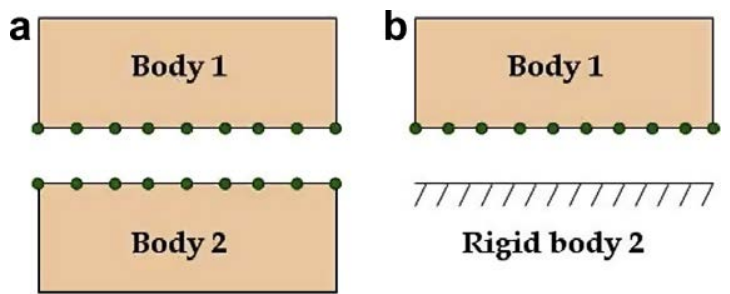

Fig. 10. Types of contact: (a) deformable; (b) rigid. 
Table 1

Different degrees of polynomials and the corresponding method used.

\begin{tabular}{|c|c|c|c|c|c|}
\hline$\alpha$ & Case number & $H\left(\sigma_{n}\right)$ & $\chi_{I}(\tau)$ & Polynomials degree & Resolution method \\
\hline$\alpha<1$ & $\mathrm{a}$ & 1 & 0 & 3 & Analytical \\
\hline$\alpha<1$ & $\mathrm{~b}$ & 0 & 1 & 3 & Analytical \\
\hline$\alpha<1$ & c & 1 & 1 & 5 & Newton \\
\hline$\alpha>1$ & $\mathrm{~d}$ & 1 & 0 & 2 & Analytical \\
\hline$\alpha>1$ & $\mathrm{e}$ & 0 & 1 & 2 & Analytical \\
\hline$\alpha>1$ & $\mathrm{f}$ & 1 & 1 & 4 & Analytical \\
\hline
\end{tabular}

Based on (20) and (17), the general expression for the damage condition can be written:

$$
\begin{aligned}
\bar{\Phi}(\alpha)= & \frac{1}{2} \bar{h} H\left(\sigma_{n}\right)\left(\frac{C_{N}}{1+h_{N} H\left(\sigma_{n}\right) \alpha}\left[u_{N}\right]\right)^{2}+\frac{1}{2} \bar{k} \chi_{I}(\tau)\left(\frac{C_{T}}{1+h_{T} \chi_{I}(\tau) \alpha}\left[u_{T}\right]\right. \\
& \left.-\frac{ \pm \mu C_{N}}{\left(1+h_{N} H\left(\sigma_{n}\right) \alpha\right)\left(1+h_{T} \chi_{I}(\tau) \alpha\right)}\left[u_{N}\right]\right)^{2}-\bar{R}(\alpha) \leqslant 0
\end{aligned}
$$

In order to satisfy (29), we must find the roots $\alpha$ of $\bar{\Phi}(\alpha)$. For this purpose:

- If $\bar{\Phi}(\alpha)$ is negative, there is no damage and the value obtained at the previous time step is kept.

- If $\bar{\Phi}(\alpha)$ is positive, the damage evolves and we must solve the equation $\bar{\Phi}(\alpha)=0$, that is, we have to find the roots of a polynomial equation. The degree of this polynomial differs depending on the values of $H\left(\sigma_{n}\right)$ and $\chi_{I}(\tau)$. This is summarized in Table 1 , along with the method used to solve the problem.

Cases $a$ and $d$ correspond to pure traction, $b$ and $e$ to pure sliding and, $c$ and $f$ to combined traction and sliding conditions.

\subsection{Algorithms}

Two incremental algorithms were used to solve the A-PL asymptotic model [17].

- The first algorithm is an implicit one with a double convergence condition: a polynomial and a fixed point method are used simultaneously to satisfy the equilibrium equation and to confirm that the damage variable $\alpha$ belongs to the validity domain $\Phi(\alpha)$, by solving the linear non-symmetric system (28) beforehand, thus updating the condensed interface matrix $\widetilde{A}_{c c}$. At each iteration, the systems are solved using the Gauss method, whereas the damage conditions are obtained using the "polynomial method". It is difficult to make sure that this algorithm converges because of the small validity domain and because the updated matrix changes at each iteration by one time increment.

- The second algorithm is a simpler one: it gives explicit solution with an unique verification that the damage variable belongs to the validity domain. The linear system is then solved; $\widetilde{A}_{c c}$ is therefore estimated once at each time increment.

\subsubsection{Implicit algorithm}

Note that the first superscript refers to the time step and the second refers to the non-linear iteration number.

$\alpha^{0}$ is given. Looking for $\alpha^{i}$ at the time step $\Delta t^{i}$, we obtain $\alpha^{i, 0}=\alpha^{i-1}$. At the iteration $n=n+1$, we perform the following steps:

- Constructing the elementary rigidity matrix $V^{e l}$ with each quadrangle.

- Constructing of the condensed matrix $\widetilde{A}_{c c}\left(\alpha^{i, n}\right)$.

- Solving the linear system (Gauss): $\left(A_{c c}+\widetilde{A}_{c c}\left(\alpha^{i, n}\right)\right) U^{i, n}=F_{c}$.
- Estimating the displacement jump $\left[u^{i, n}\right]$.

- Estimating the stress $\sigma_{i, n}=K^{i, n}\left[u^{i, n}\right]$ with $K^{i, n}$ given by (17).

- Estimating $\bar{Y}$.

- Estimating $\bar{R}\left(\alpha^{i, n}\right)$

- Testing $\bar{\Phi}\left(\alpha^{i, n}\right)=\bar{Y}-\bar{R}\left(\alpha^{i, n}\right)$ :

- If negative, then the previous value $\alpha^{i, n}$ is kept.

- If positive, then find the roots $\alpha^{i, n}$ of the polynomial $\bar{\Phi}\left(\alpha^{i, n}\right)$ using the polynomial method.

Iterate the fixed point while $\left|\bar{\Phi}\left(\alpha^{i, n}\right)\right|>10^{-4}$.

\subsubsection{Explicit algorithm}

First time step:

- $\alpha^{0}=0$ and $\left[u^{0}\right]=0$.

- Constructing the elementary rigidity matrix $V^{e l}$ in the case of each quadrangle.

- Estimating $\bar{Y}$ and $\bar{R}\left(\alpha^{0}\right)$.

- Since the jump $\left[u^{0}\right]$ is known, we can look for $\alpha^{1}$, the solution of: $\Phi\left(\alpha^{1}\right)=Y-R\left(\alpha^{1}\right)=0 \Rightarrow$ Therefore, we must find $\alpha^{1}=0$.

- Constructing the condensed matrix $\widetilde{A}_{c c}\left(\alpha^{0}\right)$.

- System solving $\left(A_{c c}+\widetilde{A}_{c c}\left(\alpha^{1}\right)\right) U^{1}=F_{c}^{1} \Rightarrow$ Deducing the displacement jump $\left[u^{1}\right]$.

Time step $i+1$ with $i \geq 2$ :

- $\left[u^{i}\right]$ is known.

- Constructing the elementary rigidity matrix $V^{e l}$ in the case of each quadrangle.

- Estimating $\bar{Y}$ and $\bar{R}\left(\alpha^{i}\right)$.

- Looking for $\alpha^{i+1}$, the solution of the polynomial $\bar{\Phi}\left(\alpha^{i+1}\right)=\bar{Y}-\bar{R}\left(\alpha^{i+1}\right)$.

- Constructing the condensed matrix $\widetilde{A}_{c c}\left(\alpha^{i}\right)$.

- Solving the system $\left(A_{c c}+\widetilde{A}_{c c}\left(\alpha^{i+1}\right)\right) U^{i+1}=F_{c}^{i+1} \Rightarrow$ Deducing the displacement jump $\left[u^{i+1}\right]$.

Comment: For both algorithms, the convergence is not rigorously proved. In practice, the explicit algorithm seems always convergent but requires small time step, whereas the implicit algorithm can fail in some cases.

\subsubsection{Implementation}

The two algorithms used to solve the A-GL model have been integrated into the Gyptis 2D software [9,11,18]. Gyptis, which was developed at the LMA, is based on the finite element method and can be used to solve various quasi-static and dynamic mechanical problems involving contact and friction.

\section{Numerical results}

\subsection{An academic test: the wrenching of a piece}

Let us study an academic wrenching test on a single rectangular piece. The piece is bonded at the bottom. The top is subjected to 


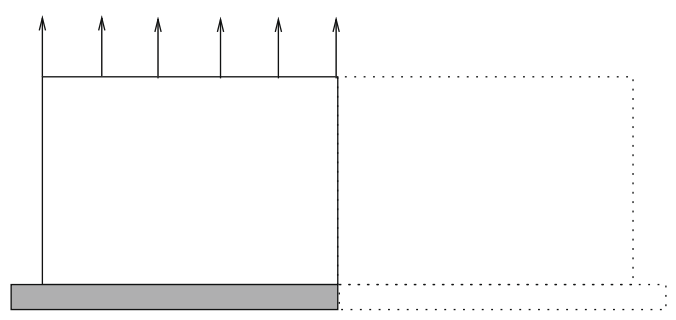

Fig. 11. Wrenching test.

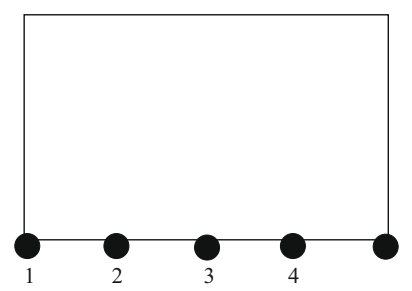

Fig. 12. Number of the four contact elements.

vertical forces. Details of this test are presented in Fig. 11. A vertical force equal to $25 \mathrm{kN}$ is applied gradually. For the sake of symmetry, the structure is embedded on its right lateral edge. The (academic) coefficients are $E=9500 \mathrm{MPa}, v=0.13, C_{N}=C_{T}=$ $5000 \mathrm{kN} \mathrm{cm}^{-3}, \mu=0.3$ (see [3]), $\bar{h}=\bar{k}=0.04 \mathrm{kN}^{-1} \mathrm{~cm}^{3}$, and $\bar{R}_{0}=$ $10 \mathrm{kN} \mathrm{cm}^{-1}$ (see [6]).

In this case, the numerical simulations are performed using the explicit algorithm and assuming the existence of a "rigid" contact interface. There are four contact elements. The number of the nodes are given in Fig. 12 and used in Figs. 13, 14, 17 and 18. The damage evolution of each component of the small wall is studied during the time simulation. Fig. 13 shows the increase in the damage during the loading time and its propagation one element after another. The damage can be seen to begin on the left of the contact zone and to propagate into the interface up to rupture.

Fig. 14 shows the evolution of the jump in the normal displacement depending on the loading conditions. We can see complete rupture of the structure occurs.

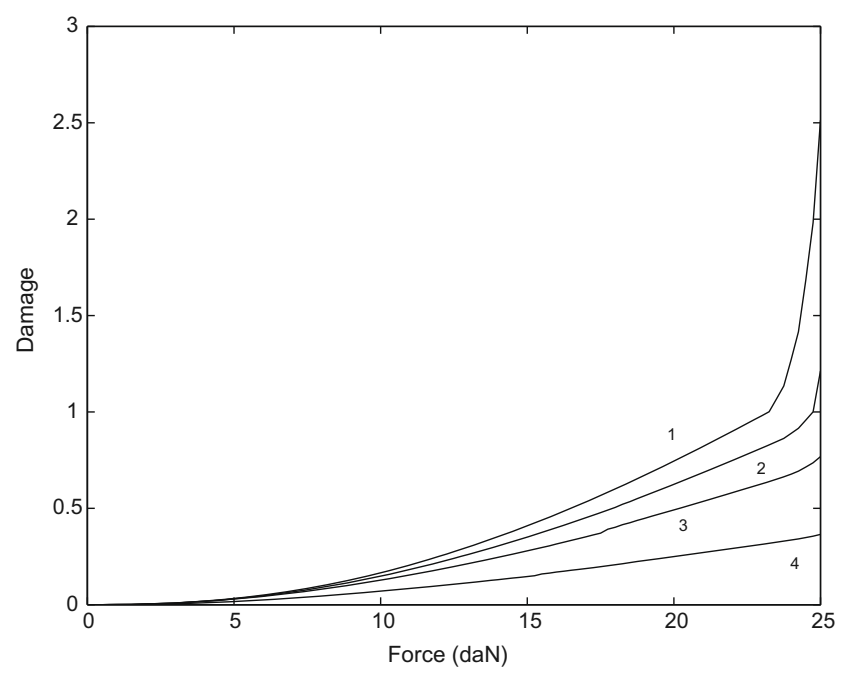

Fig. 13. Damage evolution in the case of traction test (for the four nodes).

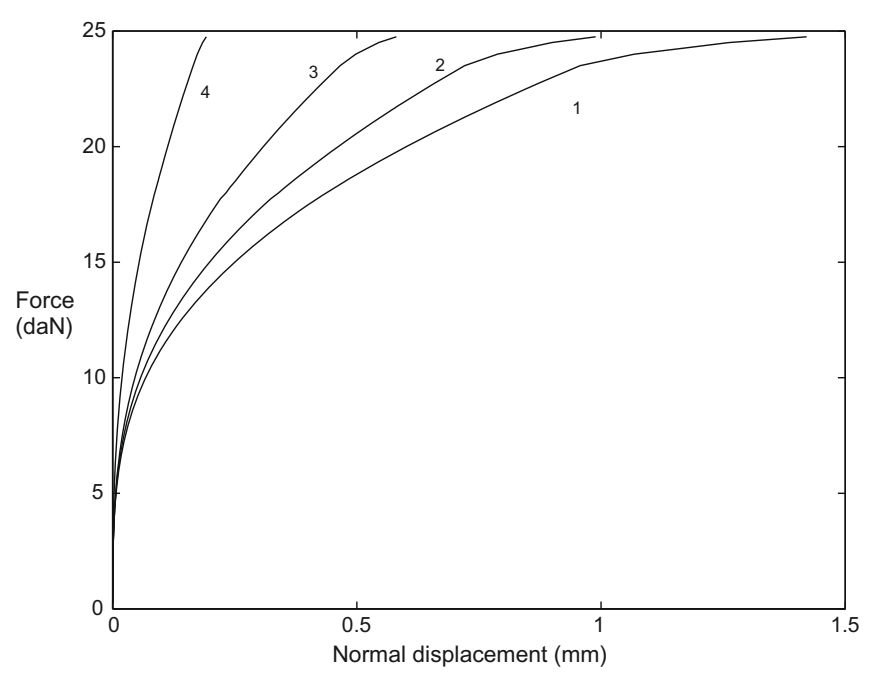

Fig. 14. Normal displacement jump evolution in the case of traction test (for the four nodes).

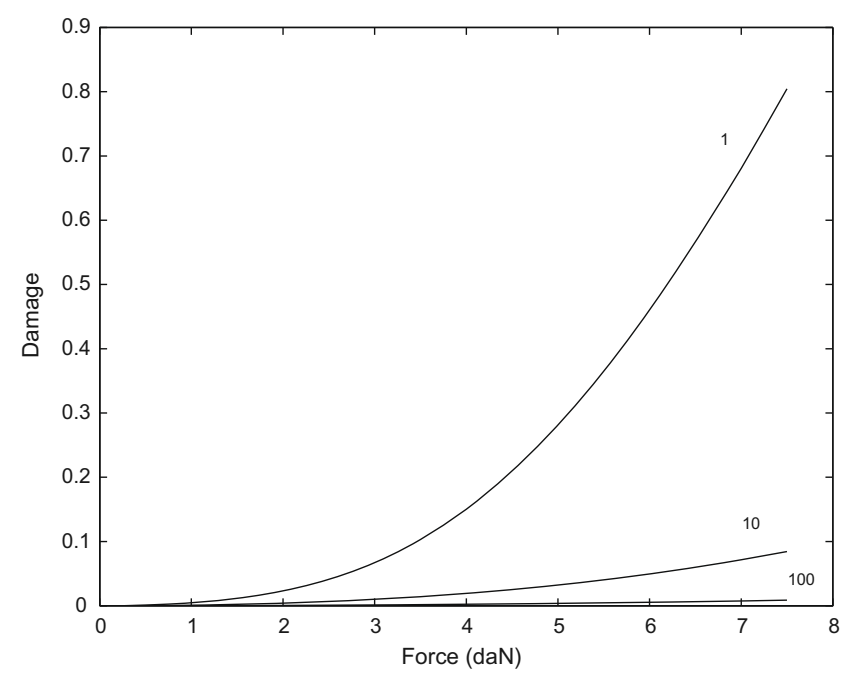

Fig. 15. Damage evolution in the case of three values of $\bar{R}_{0}$ (traction test).

Fig. 15 shows that the evolution of the damage depends strongly on the value of $\bar{R}_{0}$. As can be expected, the complete rupture of the structure is reached very quickly here at a small value of this coefficient.

\subsection{Second test: shear test on an assembly}

The structure tested here is the same as the previous one, but the loading is applied on the right and left parts. Details of this test are presented in Fig. 16. A force equal to $20 \mathrm{kN}$ is gradually applied. The mesh is the same as in the previous test.

Due to the geometry and the loading conditions, the behavior of the contact zone is highly complex. The evolution of the damage along the contact zone is shown in Fig. 17. As we can see, the evolution is strongly non-linear. In particular, the damage increases strongly in a intermediate zone which is subjected to shear and traction loads together. Note that the tangential displacement has a linear evolution during the first phase and a non-linear evolution, which results in rupture, in the second phase, as was to be expected (see Fig. 18). 


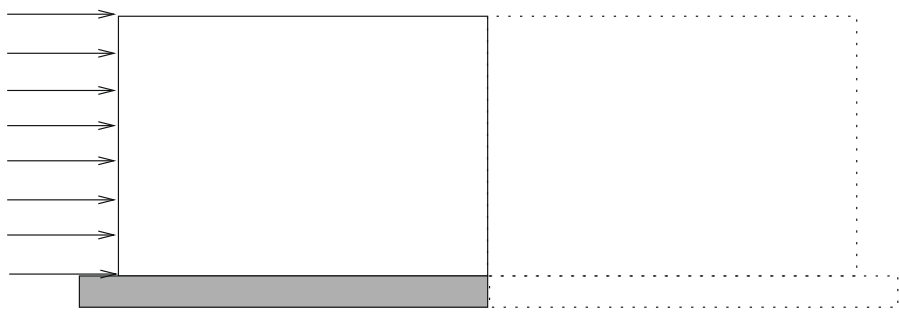

Fig. 16. Shear test.

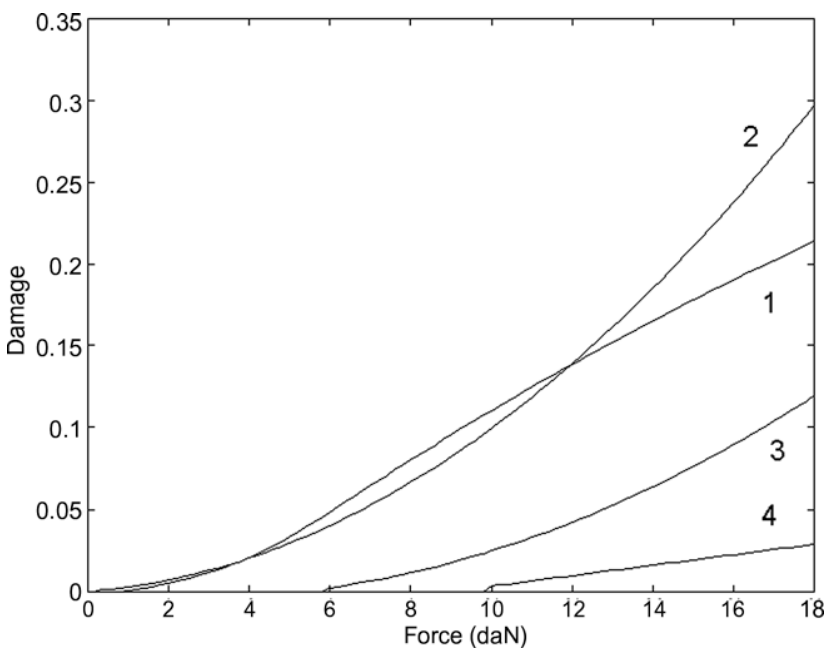

Fig. 17. Damage evolution in the case of shear test (for the four nodes).

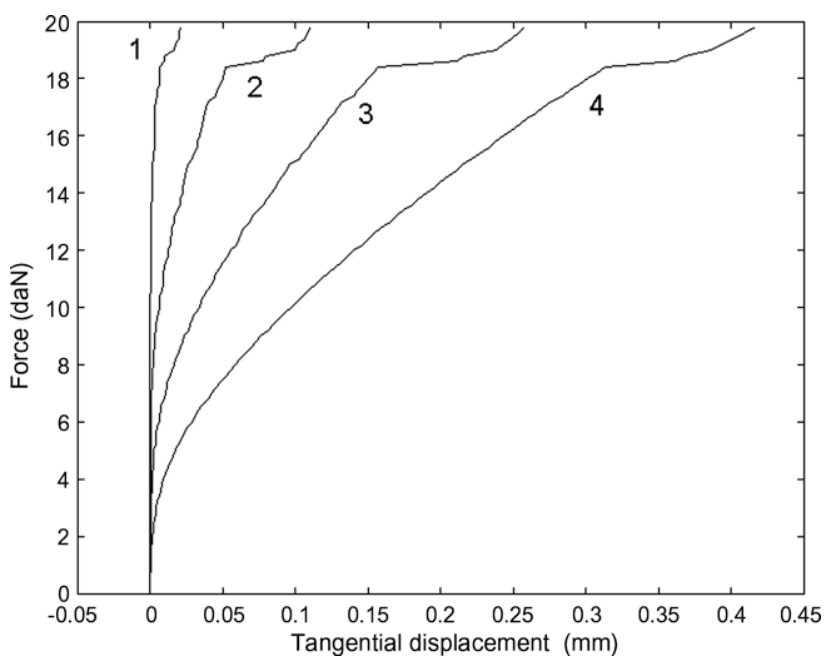

Fig. 18. Evolution of the tangential displacement (shear test).

\section{Conclusion}

Previous experimental data shown that the behavior of an interface between mortar and bricks is elastic up to rupture, and that the rupture is fragile in the case of full bricks and quasi-fragile in that of hollow bricks. In this paper, we have presented a model for studying quasi-brittle interfaces of this kind. The model is based on the study by Gambarotta and Lagomarsino [6] on masonry bricks. An asymptotic analysis was performed to obtain an interface model depending on six parameters, which relates the displacement jump non-linearly to the stress vector along the interface. The stiffness of this link depends on a damage variable, and the damage is governed by an evolution equation. Two numerical algorithms were studied. The model was implemented in the Gyptis software, and academic tests were performed to test the robustness of the algorithms. In this paper, only academic examples are presented, the results seem qualitatively in agreement with experimental data (see Fig. 3 and $[4,2]$ ).

In future studies, we intend to identify the parameters involved in the model (stiffness, damage parameters, internal friction) and it would be very interesting to provide some more complicated examples dealing with real masonry structures (for example, as done previously in [5]).

\section{References}

[1] Eckhaus W. Asymptotic analysis of singular perturbations. North-Holland; 1979.

[2] Fouchal F. Contribution à la modélisation numérique des interfaces dans les structures maçonnées. PhD thesis, Université de Reims Champagne Ardennes; 2006.

[3] Fouchal F, Lebon F, Titeux I. Contribution to the modelling of interfaces in masonry construction. Constr Build Mater 2008. doi:10.1016/j.conbuildmat. 2008.10.011.

[4] Gabor A. Contribution à la caractérisation et à la modélisation des maçonneries non-renforcées et renforcées par materiaux composites. PhD thesis, Université Claude-Bernard Lyon I; 2002.

[5] Gabor A, Bennani A, Jacquelin E, Lebon F. Modelling approaches of the in-plane shear behaviour of unreinforced and frp strengthened masonry panels. Compos Struct 2006;74:277-88.

[6] Gambarotta L, Lagomarsino S. Damage models for the seismic response of brick masonry walls. Part I: The continuum model and its application. Eartquake Eng Struct Dynam 1997;26:441-62.

[7] Geymonat G, Krasucki F. Analyse asymptotique du comportement en flexion de deux plaques collées. CR Acad Sci I 1997;325:307-14.

[8] Klarbring A. Derivation of the adhesively bonded joints by the asymptotic expansion method. Int J Eng Sci 1991;29:493-512.

[9] Latil J-C, Raous M. Elément finis et inéquations variationnelles, résolution de quelques problèmes de contact et de frottement, logiciel gyptis. Technical report, Note interne LMA; 2007.

[10] Lebon F, Ould-Khaoua A, Licht C. Numerical study of soft adhesively bonded joints in finite elasticity. Comput Mech 1997;21:134-40.

[11] Lebon F, Raous M. Friction modelling of a bolted junction under internal pressure loading. Comput Struct 1992;43:925-33.

[12] Lebon F, Rizzoni R. Asymptotic study of soft thin layer: the non-convex case. Mech Adv Mater Struct 2008;15:12-20.

[13] Lebon F, Rizzoni R, Ronel S. Analysis of non-linear soft thin interfaces. Comput Struct 2004;82:1929-38.

[14] Lebon F, Ronel S. First order numerical analysis of linear thin layers. ASME J Appl Mech 2007;74:824-8.

[15] Lebon F, Ronel-Idrissi S. Asymptotical analysis of Mohr-Coulomb and Drucker-Prager soft thin layers. Steel Compos Struct 2004;4:133-47.

[16] Licht C, Michaille G. A modeling of elastic adhesive bonded joints. Adv Math Sci Appl 1997;7:711-40.

[17] Pelissou C, Fouchal F, Lebon F, Titeux I. Damage interface models for brick masonry walls. In: Topping BHV, editor. Proceedings of the 11 th international conference on civil, structural and environmental engineering computing. Civil-Comp Press; 2007.

[18] Raous M, Chabrand P, Lebon F. Numerical methods for solving unilateral contact problem with friction. J Theor Appl Mech 1988;7:111-28.

[19] Sanchez-Hubert J, Sanchez-Palencia E. Introduction aux méthodes asymptotiques et à l'homogénisation. Masson; 1992.

[20] Wriggers P, Panagiotopoulos P. New developments in contact problems. Springer; 1999. 